\title{
molecules
}

ISSN 1420-3049

http://www.mdpi.org

Full Paper

\section{New Diterpenoid Alkaloids from the Roots of Delphinium tiantaishanense}

\author{
Jie Li, Dong-Lin Chen, Xi-Xian Jian and Feng-Peng Wang* \\ Department of Chemistry of Medicinal Natural Products, West China College of Pharmacy, Sichuan \\ University, No. 17, Duan 3, Renmin Nan Road, Chengdu 610041, P. R. China \\ * Author to whom correspondence should be addressed; E-mail: wfp@wcums.edu.cn
}

Received: 11 January 2007; in revised form: 14 February 2007 / Accepted: 18 February 2007 / Published: 8 March 2007

\begin{abstract}
Four new diterpenoid alkaloids: tiantaishansine (1), tiantaishannine (2), tiantaishanmine (3), and tiantaishandine (4) have been isolated from the roots of Delphinium tiantaishan. Their structures were elucidated by chemical evidence and spectral analyses, including ESI-MS, HR-EI-MS, 1D- and 2D-NMR.
\end{abstract}

Keywords: Dephinium tiantaishanense; tiantaishansine; tiantaishannine; tiantaishanmine; tiantaishandine

\section{Introduction}

The plant Dephinium tiantaishanense W. J. Zhang et G. H. Chen is a new species plant of Dephinium L., mainly distributed around Tiantai Mountain in Pengzhou County, Sichuan Province, China [1]. To our knowledge, no phytochemical investigation of this plant has been undertaken. In the course of our comparative studies of diterpenoid alkaloids from Aconitum and Delphinium species, four new diterpenoid alkaloids: tiantaishansine (1), tiantaishannine (2), tiantaishanmine (3), and tiantaishandine (4), were isolated from the roots of $D$. tiantaishanense. This paper describes the isolation and structure elucidation of these new alkaloids. 


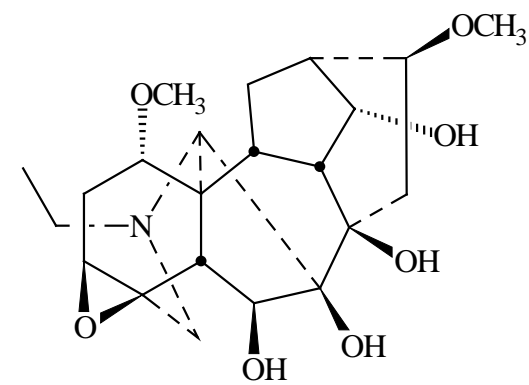

1

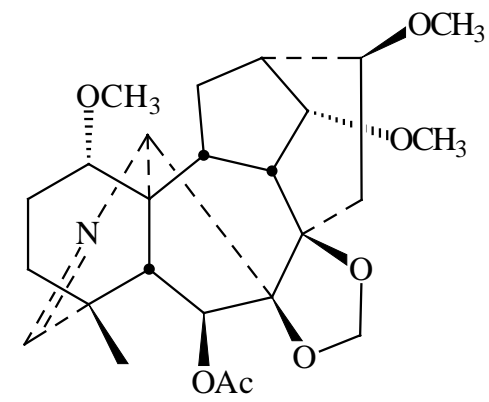

3

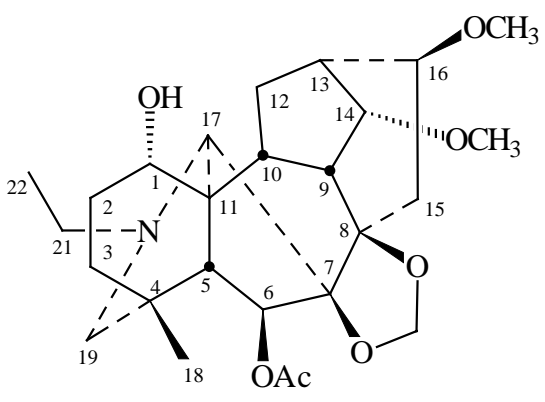

2

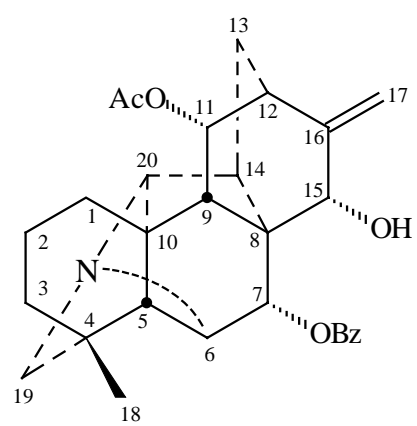

4

\section{Results and Discussion}

Tiantaishansine (1) was obtained as an amorphous powder. Its molecular formula $\mathrm{C}_{22} \mathrm{H}_{33} \mathrm{NO}_{7}$ was inferred from its HR-ESI-MS and ${ }^{13} \mathrm{C}$-NMR. Its NMR spectra displayed an $N$-ethyl group $\left(\delta_{\mathrm{H}} 1.07,3 \mathrm{H}\right.$, t, $J=7.2 \mathrm{~Hz} ; \delta_{\mathrm{C}} 49.9$ t, 13.9 q) and two methoxyl groups ( $\delta_{\mathrm{H}} 3.51,3.41$, s, each $3 \mathrm{H}_{\text {; }} \delta_{\mathrm{C}}$ see Table 1$)$. These two methoxyl groups could be assigned at $\mathrm{C}-1$ and $\mathrm{C}-16$, respectively, based on the long-range correlations between 1- $\mathrm{OCH}_{3}\left(\delta_{\mathrm{H}} 3.51\right) / \mathrm{C}-1\left(\delta_{\mathrm{C}} 77.7\right)$ and $16-\mathrm{OCH}_{3}\left(\delta_{\mathrm{H}} 3.41\right) / \mathrm{C}-16\left(\delta_{\mathrm{C}} 82.3\right)$ in HMBC spectrum (Figure 1). The ${ }^{13} \mathrm{C}$-NMR spectrum showed the 3,4-epoxy group based on the downfield signal at C-3 $\left(\delta_{\mathrm{C}} 57.8\right.$, d) and C-4 $\left(\delta_{\mathrm{C}} 58.3 \mathrm{~s}\right)$ [2]. The one-proton triplet $(J=4.4 \mathrm{~Hz})$ at $\delta_{\mathrm{H}}$ 4.03 in the ${ }^{1} \mathrm{H}-\mathrm{NMR}$ spectrum of 1 was assigned to $\mathrm{H}-14 \beta$ based on the multiplicity and the coupling constant, resulting in location of the hydroxy group at C-14 [2]. The IR $\left(3425 \mathrm{~cm}^{-1}\right)$ and ${ }^{13} \mathrm{C}-\mathrm{NMR}\left(\delta_{\mathrm{C}}\right.$ 91.3 s, 84.4 s, 79.8 d, 74.3 d) spectra showed that there were two tertiary hydroxyl groups and two secondary hydroxyl groups. Except for the secondary hydroxyl group at C-14, the other two tertiary hydroxyl groups could be attributed to C-7 and C-8 based on the HMBC correlations between 5-H/C-7 and 9-H/C-8, respectively. The remaining hydroxyl group could be located at C-6 due to the chemical shift of H-6 ( $\delta_{\mathrm{H}} 4.82 \mathrm{~s}$ ) and the long-range correlations between H-6/C-5, C-7 in the HMBC spectrum (Figure 1). Thus, on the basis of these observations, the structure of tiantaishansine was established as 1, which corresponds to a $\mathrm{C}_{18}$-diterpenoid alkaloid.

Tiantaishannine (2) was isolated as an amorphous powder, mp 206-208 ${ }^{\circ} \mathrm{C}$. Its pseudo-molecular formula, $\mathrm{C}_{26} \mathrm{H}_{40} \mathrm{NO}_{7}$, was derived from HR-ESI-MS (m/z 478.2801 $[\mathrm{M}+\mathrm{H}]^{+}$, calcd. 478.2799) and ${ }^{13} \mathrm{C}-\mathrm{NMR}$. The NMR data of compound 2 gave distinctive signals at $\delta_{\mathrm{H}} 0.99(3 \mathrm{H}, \mathrm{s}), \delta_{\mathrm{C}} 26.7 \mathrm{q}$, for an angular methyl group, $\delta_{\mathrm{H}} 1.11(3 \mathrm{H}, \mathrm{t}, J=7.2 \mathrm{~Hz}), \delta_{\mathrm{C}} 49.6 \mathrm{t}$, and $13.3 \mathrm{q}$ for the $N$-ethyl group, $\delta_{\mathrm{H}} 3.36$, 3.43 (each $3 \mathrm{H}, \mathrm{s}$ ) for two methoxyl groups, $\delta_{\mathrm{H}} 2.07(3 \mathrm{H}, \mathrm{s}), \delta_{\mathrm{C}} 21.5 \mathrm{q}$ and $169.7 \mathrm{~s}$ for an acetyl group. The signals at $\delta_{\mathrm{H}} 4.92$ and 4.96 (each $1 \mathrm{H}, \mathrm{s}$ ) in ${ }^{1} \mathrm{H}$ - NMR along with signals at $\delta_{\mathrm{C}} 84.4 \mathrm{~s}, 90.9 \mathrm{~s}$, and $93.9 \mathrm{t}$ in ${ }^{13} \mathrm{C}$-NMR showed the presence of 7,8-methylenedioxy group, indicating a lycoctonine-type 
$\mathrm{C}_{19}$-diterpenoid alkaloid [2,3]. Two methoxyl groups could be located at C-14 and C-16 due to the long-range correlations between $14-\mathrm{OCH}_{3}\left(\delta_{\mathrm{H}} 3.43 \mathrm{~s}\right)$ and $\mathrm{C}-14\left(\delta_{\mathrm{C}} 83.5 \mathrm{~d}\right), 16-\mathrm{OCH}_{3}\left(\delta_{\mathrm{H}} 3.36, \mathrm{~s}\right)$ and C-16 ( $\left.\delta_{\mathrm{C}} 82.2 \mathrm{~d}\right)$ in the HMBC spectrum of 2 (Figure 1 ), as well as the acetyl group could be located at C-6 based on the HMBC correlations between H-6 $\left(\delta_{\mathrm{H}} 5.44\right.$, s)/6- $\mathrm{OCOCH}_{3}\left(\delta_{\mathrm{C}} 169.7\right)$. The broadened singlet ( $\delta_{\mathrm{H}} 3.81$ ), which could be assigned to $\mathrm{H}-1 \beta$, in combination with the C- 1 chemical shift at $\delta_{\mathrm{C}}$ $71.6 \mathrm{~d}$, suggested a hydroxyl substitution at C-1 [2]. Therefore, the structure of taintaishannine was determined as 2 .

Figure 1 key ${ }^{1} \mathrm{H}_{-}{ }^{1} \mathrm{H}$ COSY ( $\left.~-~\right)$ and $\operatorname{HMBC}(\curvearrowright)$ ) correlations of $\mathbf{1}$ and $\mathbf{2}$
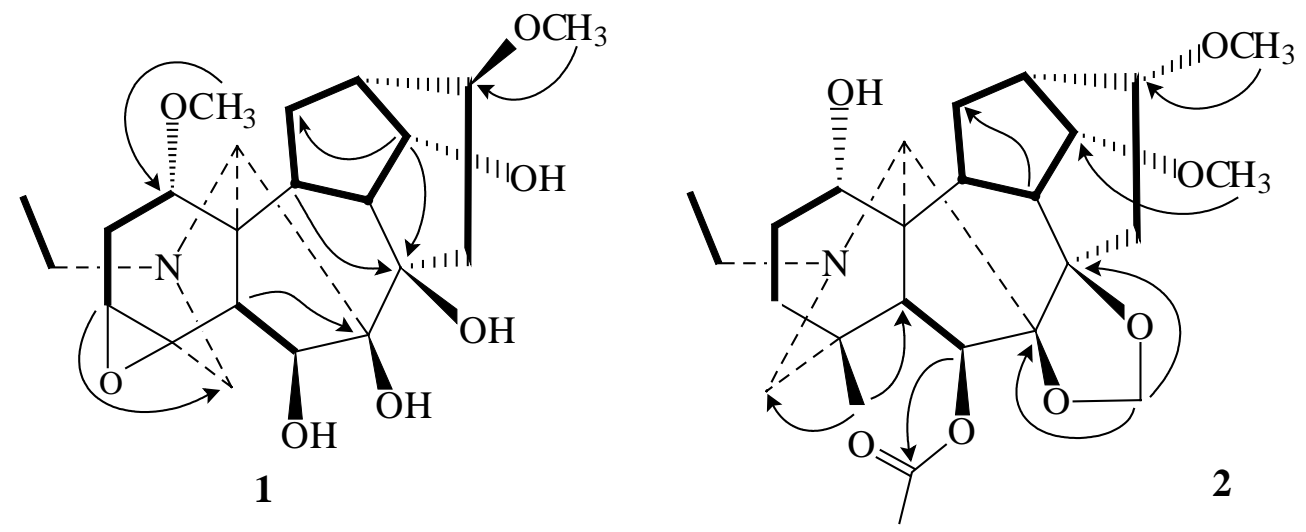

The molecular formula $\left(\mathrm{C}_{25} \mathrm{H}_{35} \mathrm{NO}_{7}\right)$ of compound 3 was determined by HR-ESI-MS $(\mathrm{m} / \mathrm{z}$ 462.2473, $\left.[\mathrm{M}+\mathrm{H}]^{+}\right)$. The NMR spectra strongly suggested that it was a lycoctonine-type diterpenoid alkaloid [2,3], with an angular methyl group $\left(\delta_{\mathrm{H}} 1.17,3 \mathrm{H}, \mathrm{s} ; \delta_{\mathrm{C}} 22.1 \mathrm{q}\right)$, three methoxyl groups $\left(\delta_{\mathrm{H}}\right.$ 3.22, 3.34, 3.45, each $3 \mathrm{H}, \mathrm{s} ; \delta_{\mathrm{C}}$ see Table 2$)$, an acetyl group $\left(\delta_{\mathrm{H}} 2.06,3 \mathrm{H}, \mathrm{s} ; \delta_{\mathrm{C}} 21.5 \mathrm{q}, 169.5 \mathrm{~s}\right)$, and a methylenedioxy group $\left(\delta_{\mathrm{H}} 4.93,4.97\right.$, each $\left.1 \mathrm{H}, \mathrm{s} ; \delta_{\mathrm{C}} 93.9 \mathrm{t}\right)$. The significant absence of $N$-methyl, $N$-ethyl showed that there are no substitutions on the nitrogen atom of $\mathbf{3}$ besides the imine moiety $\left(\delta_{\mathrm{H}}\right.$ $\left.7.46 \mathrm{br} \mathrm{s} ; \delta_{\mathrm{C}} 169.9 \mathrm{~d}\right)$. The downfield signal at $\delta_{\mathrm{C}} 44.4 \mathrm{~s}$ can be assigned to C-4 adjacent to a double bond ( $\mathrm{N}=\mathrm{C}-19)$ [4]. Three methoxyl groups could be located at $\mathrm{C}-1, \mathrm{C}-14$, and $\mathrm{C}-16$ based on the HMBC correlations of $1-\mathrm{OCH}_{3}\left(\delta_{\mathrm{H}} 3.22\right) / \mathrm{C}-1\left(\delta_{\mathrm{C}} 80.4\right), 14-\mathrm{OCH}_{3}\left(\delta_{\mathrm{H}} 3.45\right) / \mathrm{C}-14\left(\delta_{\mathrm{C}} 83.4\right), 16-\mathrm{OCH}_{3}$ $\left(\delta_{\mathrm{H}} 3.34\right) / \mathrm{C}-16\left(\delta_{\mathrm{C}} 81.3\right)$ (Figure 2$)$. The one-proton singlet at $\delta_{\mathrm{H}} 5.25 \mathrm{~s}$ was assigned to $\mathrm{H}-6 \alpha$ suggesting an acetyl substitution at C-6 due to the HMBC correlation of $\mathrm{H}-6 / \mathrm{COCH}_{3}$. All these evidence supported the structure of compound $\mathbf{3}$ and named tiantaishanmine.

Figure 2 key ${ }^{1} \mathrm{H}_{-}{ }^{1} \mathrm{H} \operatorname{COSY}(-)$ ) and $\mathrm{HMBC}(\frown$ ) correlations of $\mathbf{3}$ and $\mathbf{4}$
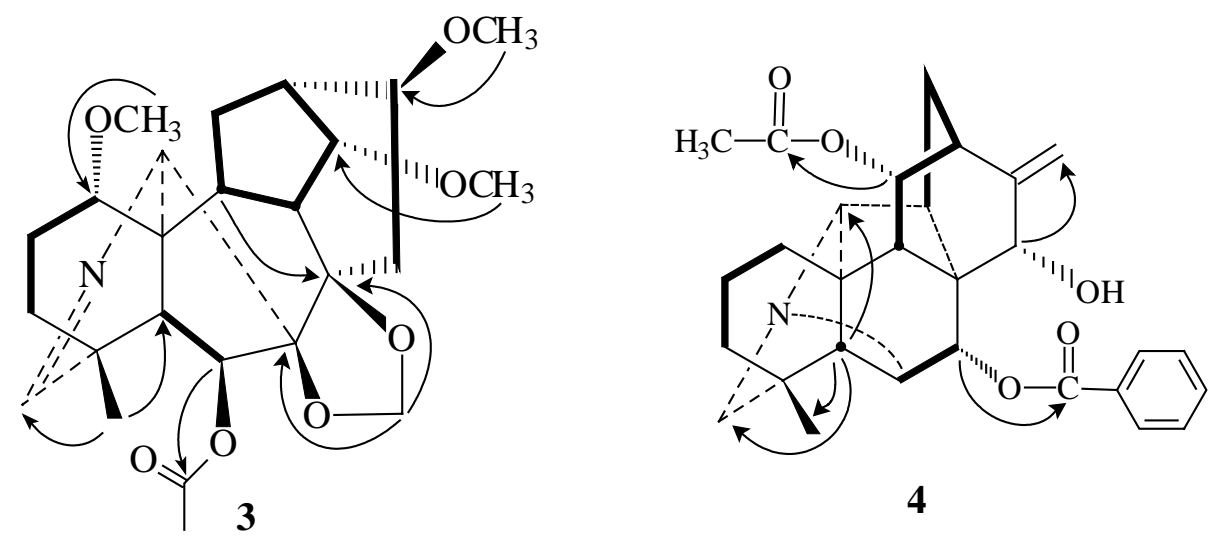
Table 1. NMR data of compounds 1 and 2 in $\mathrm{CDCl}_{3}\left(400 \mathrm{MHz}\right.$ for ${ }^{1} \mathrm{H}, 100 \mathrm{MHz}$ for ${ }^{13} \mathrm{C}$ ).

\begin{tabular}{|c|c|c|c|c|}
\hline \multirow[t]{2}{*}{ NO. } & \multicolumn{2}{|r|}{1} & \multicolumn{2}{|r|}{2} \\
\hline & $\delta_{\mathrm{C}}$ & $\begin{array}{l}\delta_{\mathrm{H}} \text { Mult } \\
(J=\mathrm{Hz})\end{array}$ & $\delta_{\mathrm{C}}$ & $\begin{array}{l}\delta_{\mathrm{H}} \text { Mult } \\
(J=\mathrm{Hz})\end{array}$ \\
\hline 1 & $77.7 \mathrm{~d}$ & $3.90 \mathrm{~s}$ & $71.6 \mathrm{~d}$ & 3.81 br s \\
\hline \multirow[t]{2}{*}{2} & $31.6 \mathrm{t}$ & $1.26 \mathrm{~m}(\alpha)$ & $29.2 \mathrm{t}$ & 1.26 br s $(\alpha)$ \\
\hline & & $2.18 \mathrm{~m}(\beta)$ & & 1.56 br s $(\beta)$ \\
\hline \multirow[t]{2}{*}{3} & $57.8 \mathrm{~d}$ & $3.05 \mathrm{~m}$ & $30.9 \mathrm{t}$ & $1.54 \mathrm{~m}(\beta)$ \\
\hline & & - & & $1.58 \mathrm{~m}(\alpha)$ \\
\hline 4 & $58.3 \mathrm{~s}$ & - & $32.8 \mathrm{~s}$ & - \\
\hline 5 & $52.2 \mathrm{~d}$ & $1.56 \mathrm{~s}$ & $51.1 \mathrm{~d}$ & $1.39 \mathrm{~m}$ \\
\hline 6 & $79.8 \mathrm{~d}$ & $4.82 \mathrm{~s}$ & $78.2 \mathrm{~d}$ & $5.44 \mathrm{~s}$ \\
\hline 7 & $91.3 \mathrm{~s}$ & - & $90.9 \mathrm{~s}$ & - \\
\hline 8 & $84.4 \mathrm{~s}$ & - & $84.4 \mathrm{~s}$ & - \\
\hline 9 & $43.0 \mathrm{~d}$ & 3.12 t (5.6) & $39.5 \mathrm{~d}$ & $3.44 \mathrm{~m}$ \\
\hline 10 & $43.5 \mathrm{~d}$ & $2.12 \mathrm{~m}$ & $45.2 \mathrm{~d}$ & $2.14 \mathrm{~m}$ \\
\hline 11 & $54.0 \mathrm{~s}$ & - & $51.3 \mathrm{~s}$ & - \\
\hline \multirow[t]{2}{*}{12} & $29.5 \mathrm{t}$ & $1.58 \mathrm{~m}(\beta)$ & $30.3 \mathrm{t}$ & $1.87 \mathrm{~m}(\beta)$ \\
\hline & & $2.12 \mathrm{~m}(\alpha)$ & & $2.18 \mathrm{~m}(\alpha)$ \\
\hline 13 & $40.4 \mathrm{~d}$ & $2.26 \mathrm{~m}$ & $37.2 \mathrm{~d}$ & $2.44 \mathrm{~m}$ \\
\hline 14 & $74.3 \mathrm{~d}$ & $4.03 \mathrm{t}(4.4)$ & $83.5 \mathrm{~d}$ & $3.73 \mathrm{t}(4.0)$ \\
\hline \multirow[t]{2}{*}{15} & $26.7 \mathrm{t}$ & $1.97 \mathrm{~m}(\alpha)$ & $34.2 \mathrm{t}$ & $1.88 \mathrm{~m}(\alpha)$ \\
\hline & & $2.59 \mathrm{~m}(\beta)$ & & $2.51 \mathrm{~m}(\beta)$ \\
\hline 16 & $82.3 \mathrm{~d}$ & $3.39 \mathrm{~s}$ & $82.2 \mathrm{~d}$ & $3.28 \mathrm{~m}$ \\
\hline 17 & $67.2 \mathrm{~d}$ & $2.91 \mathrm{~d}(1.5)$ & $65.3 \mathrm{~d}$ & $3.02 \mathrm{~s}$ \\
\hline 18 & - & - & $26.7 \mathrm{q}$ & $0.99 \mathrm{~s}$ \\
\hline \multirow[t]{2}{*}{19} & $54.3 \mathrm{t}$ & $2.49 \mathrm{ABq}$ & $61.1 \mathrm{t}$ & $2.44 \mathrm{~m}$ \\
\hline & - & 3.36 ABq hidden & & $2.51 \mathrm{~m}$ \\
\hline \multirow[t]{2}{*}{21} & $49.9 \mathrm{t}$ & $2.98 \mathrm{~m}$ & $49.6 \mathrm{t}$ & $2.74 \mathrm{~m}$ \\
\hline & & $3.39 \mathrm{~m}$ & & $2.87 \mathrm{~m}$ \\
\hline 22 & $13.9 \mathrm{q}$ & $1.07 \mathrm{t}(7.2)$ & 13.3 q & $1.11 \mathrm{t}(7.2)$ \\
\hline $1-\mathrm{OCH}_{3}$ & $51.7 \mathrm{q}$ & $3.51 \mathrm{~s}$ & - & - \\
\hline $14-\mathrm{OCH}_{3}$ & - & & $57.4 \mathrm{q}$ & $3.43 \mathrm{~s}$ \\
\hline $16-\mathrm{OCH}_{3}$ & $56.4 \mathrm{q}$ & $3.41 \mathrm{~s}$ & $56.1 \mathrm{q}$ & $3.36 \mathrm{~s}$ \\
\hline \multirow[t]{2}{*}{$-\mathrm{OCH}_{2} \mathrm{O}-$} & & & $93.9 \mathrm{t}$ & $4.92 \mathrm{~s}$ \\
\hline & & & & $4.96 \mathrm{~s}$ \\
\hline \multirow[t]{2}{*}{ OAc } & & & $169.7 \mathrm{~s}$ & \\
\hline & & & $21.5 \mathrm{q}$ & $2.07 \mathrm{~s}$ \\
\hline
\end{tabular}


Table 2. NMR data of compounds $3\left(600 \mathrm{MHz}\right.$ for ${ }^{1} \mathrm{H}, 150 \mathrm{MHz}$ for ${ }^{13} \mathrm{C}$ ) and 4 (400 $\mathrm{MHz}$ for ${ }^{1} \mathrm{H}, 100 \mathrm{MHz}$ for ${ }^{13} \mathrm{C}$ ) in $\mathrm{CDCl}_{3}$.

\begin{tabular}{|c|c|c|c|c|}
\hline \multirow[t]{2}{*}{ NO. } & \multicolumn{2}{|r|}{3} & \multicolumn{2}{|r|}{4} \\
\hline & $\delta_{\mathrm{C}}$ & $\delta_{\mathrm{H}} \operatorname{Mult}(J=\mathrm{Hz})$ & $\delta_{\mathrm{C}}$ & $\delta_{\mathrm{H}} \operatorname{Mult}(J=\mathrm{Hz})$ \\
\hline \multirow[t]{2}{*}{1} & $80.4 \mathrm{~d}$ & $3.23 \mathrm{~m}$ & $29.0 \mathrm{t}$ & $1.55 \mathrm{~m}(\beta)$ \\
\hline & & - & & $1.81 \mathrm{~m}(\alpha)$ \\
\hline \multirow[t]{2}{*}{2} & $25.6 \mathrm{t}$ & $1.53 \mathrm{~m}(\alpha)$ & $19.9 \mathrm{t}$ & 0.87 br s $(\beta)$ \\
\hline & & $1.92 \mathrm{~m}(\beta)$ & & $1.61 \mathrm{~m}(\alpha)$ \\
\hline \multirow[t]{2}{*}{3} & $31.9 \mathrm{t}$ & $1.31 \mathrm{~m}(\beta)$ & $33.1 \mathrm{t}$ & $1.25 \mathrm{~m}$ \\
\hline & & $1.72 \mathrm{~m}(\alpha)$ & & $1.47 \mathrm{~m}$ \\
\hline 4 & $44.4 \mathrm{~s}$ & - & $37.5 \mathrm{~s}$ & - \\
\hline 5 & $52.5 \mathrm{~d}$ & $1.41 \mathrm{br} \mathrm{s}$ & $60.0 \mathrm{~d}$ & $1.67 \mathrm{~s}$ \\
\hline 6 & $79.9 \mathrm{~d}$ & $5.25 \mathrm{~s}$ & $68.0 \mathrm{~d}$ & $3.33 \mathrm{~s}$ \\
\hline 7 & $90.9 \mathrm{~s}$ & - & $70.4 \mathrm{~d}$ & $5.44 \mathrm{~d}(2.8)$ \\
\hline 8 & $82.9 \mathrm{~s}$ & - & $52.5 \mathrm{~s}$ & - \\
\hline 9 & $39.8 \mathrm{~d}$ & $3.40 \mathrm{~m}$ & $47.1 \mathrm{~d}$ & $2.43 \mathrm{dd}(6.6,1.5)$ \\
\hline 10 & $47.9 \mathrm{~d}$ & $2.20 \mathrm{~m}$ & $50.9 \mathrm{~s}$ & - \\
\hline 11 & $49.8 \mathrm{~s}$ & - & $75.4 \mathrm{~d}$ & $5.28 \mathrm{~d}(6.4)$ \\
\hline \multirow[t]{2}{*}{12} & $28.7 \mathrm{t}$ & $2.13 \mathrm{~m}(\beta)$ & $40.1 \mathrm{~d}$ & 2.28 br s \\
\hline & & $1.88 \mathrm{dd}(\alpha)(10,5.2)$ & & - \\
\hline \multirow[t]{2}{*}{13} & $38.7 \mathrm{~d}$ & $2.40 \mathrm{~m}$ & $28.0 \mathrm{t}$ & $1.43 \mathrm{~m}$ \\
\hline & & & & 2.34 m (hidden) \\
\hline 14 & $83.4 \mathrm{~d}$ & 3.73 t (3.2) & $39.2 \mathrm{~d}$ & 2.34 t (9.6) \\
\hline \multirow[t]{2}{*}{15} & $33.4 \mathrm{t}$ & $1.85 \mathrm{~m}(\alpha)$ & $66.0 \mathrm{~d}$ & $4.06 \mathrm{~s}$ \\
\hline & & $2.71 \mathrm{~m}(\beta)$ & & - \\
\hline 16 & $81.3 \mathrm{~d}$ & $3.28 \mathrm{t}(5.2)$ & $150.8 \mathrm{~s}$ & - \\
\hline 17 & $63.5 \mathrm{~d}$ & 4.16 br s & $112.2 \mathrm{t}$ & $5.04 \mathrm{~d}(0.9)$ \\
\hline 18 & $22.1 \mathrm{q}$ & $1.17 \mathrm{~s}$ & 28.9 q & $0.97 \mathrm{~s}$ \\
\hline 19 & $169.9 \mathrm{~d}$ & 7.46 br s & $62.7 \mathrm{t}$ & $2.49 \mathrm{~s}$ \\
\hline 20 & - & - & $74.1 \mathrm{~d}$ & $2.75 \mathrm{~s}$ \\
\hline $1-\mathrm{OCH}_{3}$ & $55.4 \mathrm{q}$ & $3.22 \mathrm{~s}$ & - & - \\
\hline $14-\mathrm{OCH}_{3}$ & $57.7 \mathrm{q}$ & $3.45 \mathrm{~s}$ & - & - \\
\hline $16-\mathrm{OCH}_{3}$ & $56.4 \mathrm{q}$ & $3.34 \mathrm{~s}$ & - & - \\
\hline \multirow[t]{2}{*}{$-\mathrm{OCH}_{2} \mathrm{O}-$} & 93.9 t & $4.93 \mathrm{~s}$ & - & - \\
\hline & & $4.97 \mathrm{~s}$ & & - \\
\hline \multirow[t]{2}{*}{ OAc } & $169.5 \mathrm{~s}$ & - & $170.3 \mathrm{~s}$ & - \\
\hline & $21.5 \mathrm{q}$ & $2.06 \mathrm{~s}$ & $21.4 \mathrm{q}$ & $2.06 \mathrm{~s}$ \\
\hline $\mathrm{ArCO}$ & & & $167.1 \mathrm{~s}$ & - \\
\hline $1^{\prime}$ & & & $129.7 \mathrm{~s}$ & - \\
\hline 2', 6’ & & & $130.1 \mathrm{~d}$ & 8.14 d (6.8) \\
\hline $3^{\prime}, 5^{\prime}$ & & & $128.4 \mathrm{~d}$ & $7.45 \mathrm{~m}$ \\
\hline $4^{\prime}$ & & & $133.4 \mathrm{~d}$ & $7.58 \mathrm{~m}$ \\
\hline
\end{tabular}


Tiantaishandine (4) $\mathrm{mp} 248-249{ }^{\circ} \mathrm{C}$, was obtained as an amorphous powder. The HR-ESIMS at $\mathrm{m} / \mathrm{z}$ 476.2439 corresponded to the pseudo-molecular ion $[\mathrm{M}+\mathrm{H}]^{+}\left(\mathrm{C}_{29} \mathrm{H}_{34} \mathrm{NO}_{5}\right.$ calc. 476.2431). In the NMR spectrum, some characteristic signals of a $\mathrm{C}_{20}$-diterpene alkaloid having a hetisine-type skeleton [C-4 (37.5 s), C-8 (52.5 s), C-10 (50.9, s), C-16 (150.8 s)] [5], together with some important proton signals $\left[\mathrm{H}_{3}-18\left(\delta_{\mathrm{H}} 0.97, \mathrm{~s}\right), \mathrm{H}_{2}-17\left(\delta_{\mathrm{H}} 5.04, \mathrm{~d}, J=0.9 \mathrm{~Hz}\right), \mathrm{H}-6\left(\delta_{\mathrm{H}} 3.33,1 \mathrm{H}, \mathrm{s}\right), \mathrm{H}-20\left(\delta_{\mathrm{H}} 2.75,1 \mathrm{H}, \mathrm{s}\right)\right.$, and $\left.\mathrm{H}_{2}-19\left(\delta_{\mathrm{H}} 2.49, \mathrm{~s}\right)\right]$, were observed. In addition to these data, two downfield singlets at $\delta_{\mathrm{C}} 170.3 \mathrm{~s}$, $167.1 \mathrm{~s}$, and the IR absorptions at 1736 and $1714 \mathrm{~cm}^{-1}$, showed the presence of one acetyl and one benzoyl group in this compound. The one-proton signal $\left(\delta_{\mathrm{H}} 5.28, \mathrm{~d}, J=6.4 \mathrm{~Hz}\right)$ could be attributed to $\mathrm{H}-11$, based on the HMBC corrections of $\mathrm{H}-11\left(\delta_{\mathrm{H}} 5.28\right)$ and C-9 $\left(\delta_{\mathrm{C}} 47.1\right), \mathrm{C}-10\left(\delta_{\mathrm{C}} 50.9\right), \mathrm{C}-12\left(\delta_{\mathrm{C}}\right.$ $40.1)$, as well as $1 \mathrm{H}$-doublet at $\delta_{\mathrm{H}} 5.44$, assigned to $\mathrm{H}-7$ due to the correlations of $\mathrm{H}-7\left(\delta_{\mathrm{H}} 5.44\right)$ and $\mathrm{C}-8\left(\delta_{\mathrm{C}} 52.5\right), \mathrm{C}-14\left(\delta_{. \mathrm{C}} 39.2\right), \mathrm{C}-15\left(\delta_{. \mathrm{C}} 66.0\right)$. The acetyl and benzyl groups could be located at C-11 and C-7, respectively, based on the correlations between $\mathrm{H}-11\left(\delta_{\mathrm{H}} 5.28\right) / 11-\mathrm{OCOCH}_{3}\left(\delta_{\mathrm{C}} 170.3\right)$ and $\mathrm{H}-7\left(\delta_{\mathrm{H}} 5.44\right) / 7-\mathrm{PhCO}\left(\delta_{\mathrm{C}} 167.1\right)$. The ${ }^{13} \mathrm{C}-\mathrm{NMR}$ spectrum of compound 4 displayed three oxygenated carbon signals ( $\delta_{\mathrm{C}} 66.0 \mathrm{~d}, 70.4 \mathrm{~d}, 75.4 \mathrm{~d}$ ), suggesting the presence of additional hydroxyl group, which could be placed at C-15 based on the HMBC spectrum (Figure 2). The configuration of this hydroxyl group was determined as $15 \alpha-\mathrm{OH}$, based on the NOESY relationships of $\mathrm{H}-15\left(\delta_{\mathrm{H}} 4.06\right)$ with $\mathrm{H}-17\left(\delta_{\mathrm{H}}\right.$ 5.04), $\mathrm{H}-9\left(\delta_{\mathrm{H}} 2.43\right)$ and $\mathrm{H}-12\left(\delta_{\mathrm{H}} 2.28\right)$ (Figure 3). Meanwhile the 11-OAc and 7-OBz were also assigned $\alpha$-configurations due to the NOESY experiments (Figure 3). Consequently, the structure of tiantaishandine was determined as 4 .

Figure 3. Key NOESY correlations of 4.

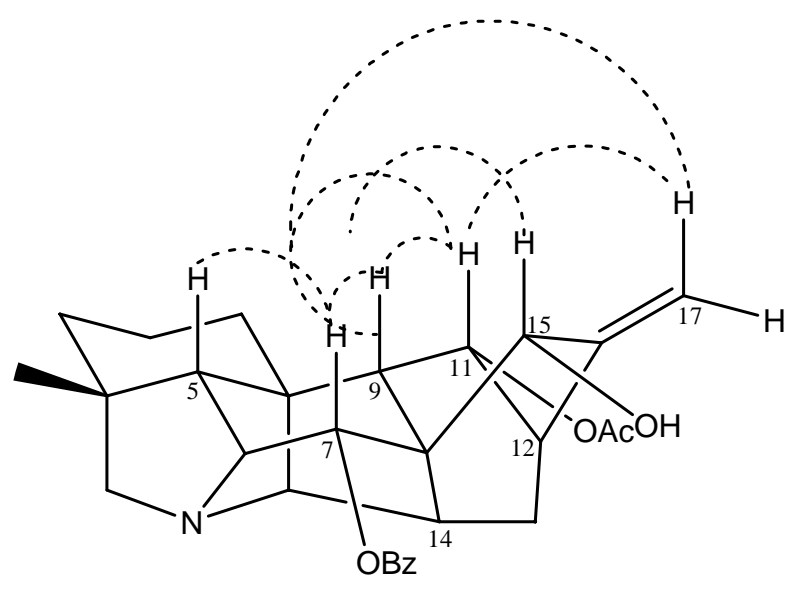

\section{Experimental}

\section{General}

Melting points were measured on a Thermal Values analytical microscope and were uncorrected. Optical rotations were recorded on a Perkin-Elmer 341 polarimeter. IR spectra were recorded on a Nicolet FI-IR 200SXY spectrophotomer. ${ }^{1} \mathrm{H}$ - and ${ }^{13} \mathrm{C}$-NMR spectra were measured in $\mathrm{CDCl}_{3}$ with TMS as the internal standard on a Varian Unity INOVA 400/54 NMR or Bruker AV-600 spectrometers. HR-ESI-MS were measured by a VG AutoSpec 3000 instrument. Silica gel GF254 and H (Qingdao Sea Chemical Factory, China) were used for TLC and column chromatography, respectively. Spots on TLC were detected with modified Dragendorff ’s reagent. 


\section{Plant Material}

Delphinium tiantaishanense W. J. Zhang et G. H. Chen was collected in the Tiantaishan mountains of Sichuan Province, P.R. China. The plant was identified taxonomically by Wen-Jing Zhang (Disease Prevention and Control Center of Pengzhou City). A voucher speciman (No. 20050818) was deposited in the West China College of Pharmacy, Sichuan University.

\section{Extraction and Isolation}

Air-dried powered roots $(4.4 \mathrm{~kg})$ of $D$. tiantaishan were percolated with $0.1 \mathrm{M} \mathrm{HCl}(50 \mathrm{~L})$. The filtrate was then made alkaline to $\mathrm{pH}>9$ with $28 \%$ aqueous $\mathrm{NH}_{4} \mathrm{OH}(1.5 \mathrm{~L})$ and extracted five times with ethyl acetate (each $20 \mathrm{~L}$ ), and the extracts evaporated to give the total crude alkaloids (58 g, yield 1.31\%). The crude alkaloids (58 g) were chromatographed over a silica gel (500 g) column eluting with a $7: 1 \rightarrow 1: 1$ petroleum ether-acetone gradient to give fractions A (7.5 g), B (9.6 g), C (18.7 g), and D (20.4 g). Fraction A (7.5 g) was rechromatographed on a silica gel column eluting with petroleum ether-acetone (8:1) to afford 2 (120 mg, yield 0.003\%). Fraction B (9.6 g) was rechromatographed on a silica gel column eluting with petroleum ether-acetone (5:1) gave 4 (90 mg, yield 0.002\%). Fraction C (18.7 g) was rechromatographed on a silica gel column eluting with petroleum-acetone (3:1) to afford 1 (100 mg, yield 0.002\%) and 3 (40 mg, yield 0.001\%), respectively.

Tiantaishansine (1). White amorphous powder, mp 94-96 ${ }^{\circ} \mathrm{C} ;[\alpha]_{D}^{20}+35.4^{\circ}\left(\mathrm{c}=0.84, \mathrm{CHCl}_{3}\right)$; $\mathrm{IR}(\mathrm{KBr})$ $\mathrm{cm}^{-1}$ : 3425, 2943, 2879, 1707, 1638, 1460; ${ }^{1} \mathrm{H}-\mathrm{NMR}$ (400 MHz, $\mathrm{CDCl}_{3}$ ) and ${ }^{13} \mathrm{C}-\mathrm{NMR}(100 \mathrm{MHz}$, $\mathrm{CDCl}_{3}$ ): see Table 1; HR-ESI-MS: $\mathrm{m} / \mathrm{z}[\mathrm{M}+\mathrm{H}]^{+}$424.2351, calcd for $\mathrm{C}_{22} \mathrm{H}_{34} \mathrm{NO}_{7}, 424.2330$.

Tiantaishannine (2). White amorphous powder, mp 206-208 ${ }^{\circ} \mathrm{C}$; $[\alpha]_{D}^{20}-23.9^{\circ}\left(\mathrm{c}=0.73, \mathrm{CHCl}_{3}\right.$ ); IR $(\mathrm{KBr}) \mathrm{cm}^{-1}$ : 3396, 2932, 1738, 1457, 1367, 1084; ${ }^{1} \mathrm{H}-\mathrm{NMR}$ (400 MHz, $\mathrm{CDCl}_{3}$ ) and ${ }^{13} \mathrm{C}-\mathrm{NMR}(100$ $\mathrm{MHz}, \mathrm{CDCl}_{3}$ ): see Table 1; HR-ESI-MS: $\mathrm{m} / \mathrm{z} 478.2801[\mathrm{M}+\mathrm{H}]^{+}$, calcd for $\mathrm{C}_{26} \mathrm{H}_{40} \mathrm{NO}_{7}, 478.2799$.

Tiantaishanmine (3). White amorphous powder, mp 251-253 ${ }^{\circ} \mathrm{C}$; $[\alpha]_{D}^{20}+24.2^{\circ}$ (c=0.26, $\mathrm{CHCl}_{3}$ ); IR $(\mathrm{KBr}) \mathrm{cm}^{-1}$ : 3396, 2926, 1741, 1637, 1461, 1365; ${ }^{1} \mathrm{H}-\mathrm{NMR}\left(600 \mathrm{MHz}, \mathrm{CDCl}_{3}\right)$ and ${ }^{13} \mathrm{C}-\mathrm{NMR}(150$ $\mathrm{MHz}, \mathrm{CDCl}_{3}$ ): see Table 2; HR-ESI-MS: $\mathrm{m} / \mathrm{z} 462.2473[\mathrm{M}+\mathrm{H}]^{+}$, calcd for $\mathrm{C}_{25} \mathrm{H}_{36} \mathrm{NO}_{7}, 462.2486$.

Tiantaishandine (4). White amorphous powder, mp 248-249 ${ }^{\circ} \mathrm{C} ;[\alpha]_{D}^{20}+35.6^{\circ}\left(\mathrm{c}=0.85, \mathrm{CHCl}_{3}\right.$ ); IR $(\mathrm{KBr}) \mathrm{cm}^{-1}$ : 3446, 2943, 1736, 1714, 1243, 1108; ${ }^{1} \mathrm{H}-\mathrm{NMR}$ (400 $\left.\mathrm{MHz}, \mathrm{CDCl}_{3}\right)$ and ${ }^{13} \mathrm{C}-\mathrm{NMR}(100$ $\mathrm{MHz}, \mathrm{CDCl}_{3}$ ): see Table 2; HR-ESI-MS: $\mathrm{m} / \mathrm{z} 476.2439[\mathrm{M}+\mathrm{H}]^{+}$, calcd for $\mathrm{C}_{29} \mathrm{H}_{34} \mathrm{NO}_{5}, 476.2431$.

\section{Acknowledgements}

This work was supported by the National Science Foundation of China (No. 30472075) 


\section{References}

1. Zhang, W. J.; Chen, G. H. A new species of Delphinium L. from Sichuan, China. West China J. Pharm Sci, 2006, 21, 560-561.

2. Pelletier, S. W.; Mody, N. V.; Joshi, B. S.; Schramn, L. C. Alkaloids: Chemical and Biological Perspectives; Pelletier S. W., ed.; John Wiley. \& Sons Press: New York, 1984; Vol. 2, pp. 206-210.

3. Pelletier, S. W.; Joshi, B. S. Alkaloids: Chemical and Biological Perspectives; Pelletier S. W., ed.; John Wiley \& Sons Press: New York, 1991; Vol. 7, pp. 297.

4. Boido, V.; Edwards, O. E.; Handa, K. L.; Kolt, R. J.; Purushothaman, K. K. Alkaloids of Acontitum columbianum Nutt. Can. J. Chem, 1984, 62, 778-784.

5. Wang, F. P.; Liang, X. T. Alkaloids: Chemistry and Biology; Cordell G. A., ed.; Elsevier Press: New York, 2002; Vol. 59, pp. 1-280.

Sample Availability: Available from the authors.

(C) 2007 by MDPI (http://www.mdpi.org). Reproduction is permitted for noncommercial purposes. 